\title{
Estresse ocupacional e presenteísmo entre enfermeiros de um hospital público
}

\author{
Occupational stress and presenteeism among nurses in a public \\ hospital
}

\author{
1 Luíza Barbosa Couto luiza.coutto1014@gmail.com \\ 2 Carolina Magalhães dos Santos \\ 3 Thaís Aparecida de Castro Palermo
}

\footnotetext{
Graduanda do curso de Enfermagem dos Institutos Superiores de Ensino do Centro Educacional Nossa Senhora Auxiliadora - ISECENSA.

2 Bióloga. Doutora em Ciências pela Fundação Oswaldo Cruz/Fiocruz. Professora e pesquisadora do Laboratório de Estudos em Saúde Pública - LAESP - dos Institutos Superiores de Ensino do Centro Educacional Nossa Senhora Auxiliadora - ISECENSA.

3 Enfermeira. Mestre em Enfermagem pela Escola de Enfermagem Anna Nery/UFRJ. Professora e pesquisadora do Laboratório de Estudos em Saúde Pública - LAESP - dos Institutos Superiores de Ensino do Centro Educacional Nossa Senhora Auxiliadora - ISECENSA.
}

\section{RESUMO}

O estudo investigou o estresse ocupacional e o presenteísmo entre enfermeiros de um hospital público. Trata-se de uma pesquisa quantitativa, descritiva, transversal, realizada nos meses de junho a setembro de 2019, com 42 enfermeiros hospitalares. Foram utilizados o Inventário de Estresse em Enfermeiros e o Questionário de Limitações no Trabalho. A maioria dos enfermeiros $(92,7 \%)$ apresentou alto estresse e um decréscimo na produtividade $(35,9 \%)$. A dimensão relações interpessoais foi influente para o alto nível de estresse ocupacional e o domínio demanda mental-interpessoal foi limitante para a produtividade desses profissionais. Conclui-se que o estresse influencia inversamente a produtividade do enfermeiro, a qualidade de vida no trabalho e a saúde desses profissionais, bem como a segurança e qualidade da assistência prestada.

\section{Palavras-chave:}

Estresse ocupacional. Presenteísmo. Enfermeiro. Saúde do trabalhador.

\begin{abstract}
The study investigated occupational stress and presenteeism among nurses in a public hospital. This is a quantitative, descriptive, cross-sectional survey, conducted from June to September 2019, with 42 hospital nurses. The Nurse Stress Inventory and the Work Limitations Questionnaire were used. Most nurses (92.7\%) had high stress and a decrease in productivity (35.9\%). The interpersonal relations dimension was influential for the high level of occupational stress and the domain mental-interpersonal demand was limiting for the productivity of these professionals. It is concluded that stress inversely influences nurses' productivity, the quality of life at work and the health of the professional, as well as the safety and quality of the assistance provided.
\end{abstract}

\section{Keywords:}

Occupational stress. Presenteeism. Nurse. Occupational health.

\section{Como você deve citar?}

COUTO, Luíza Barbosa; SANTOS, Carolina Magalhães dos; PALERMO, Thaís Aparecida de Castro . Estresse ocupacional e presenteísmo entre enfermeiros de um hospital público. Cadernos UniFOA, Volta Redonda, n. 45, p. 157-166, abril 2021. 


\section{INTRODUÇÃO}

Na sociedade moderna, o trabalho tem assumido um papel cada vez mais importante na vida dos indivíduos. Tal constatação se dá pela sua capacidade de oferecer às pessoas elementos como: renda, chance de crescimento pessoal, identidade social e autoestima. Todavia, ele pode exercer influência negativa na saúde, gerando estresse e expondo o trabalhador ao estresse ocupacional (SANTOS et al., 2017). Sintomas físicos ou mentais, resultantes de acontecimentos no ambiente laboral e/ou nas atividades estão relacionados a essa condição. Além disso, o estresse pode estar associado às situações que desestruturam o trabalhador (UENO et al., 2017).

Dentre as atividades ocupacionais com a maior possibilidade de estresse, destacam-se os profissionais da área da saúde, sendo os enfermeiros apontados como os mais afetados, por desenvolverem atividades complexas e desgastantes, exigindo desses profissionais esforço físico, mental, psicológico e emocional na implementação do seu processo de trabalho. Essas atividades podem resultar em exaustão, aumentando o estresse e interferindo na qualidade de vida e de saúde do trabalhador (MACHADO et al., 2018). Considerando a instituição hospitalar, a enfermagem é uma das profissões mais expostas ao estresse e ao adoecimento, visto que trabalha sob condições inadequadas, ambientes insalubres, com repetição de tarefas e sobrecarga de trabalho (FELIX et al., 2017).

O estresse ocupacional acontece em decorrência da forma como se lida com as necessidades do trabalho e o seu modo de enfrentamento. Várias são as fontes capazes de gerar estresse e interferir no nível de estresse do indivíduo (UENO et al., 2017). Diversas estratégias de enfretamento podem ser utilizadas para suportar as situações estressantes com vistas a possibilitar uma melhor vivência, evitando o adoecimento. Para isso, é fundamental que os profissionais reconheçam os agentes estressores presentes no ambiente laboral (OLIVEIRA et al., 2017).

Além das inúmeras consequências à saúde mental e física do profissional exposto de forma contínua ao estresse no trabalho, alguns casos podem resultar em um fenômeno conhecido como presenteísmo (CUNHA; BALSANELLI, 2015). O presenteísmo tem sido abordado de distintas formas na literatura científica, entretanto duas principais abordagens têm sido usadas com maior frequência. A primeira sugere uma abordagem mais geral, considerando vários aspectos relevantes para o fenômeno de assistir ao trabalho como doente. E a segunda, foca na perda da produtividade decorrente do trabalho prestado em condição de adoecimento (SKAGEN; COLLINS, 2016).

Embora bastante frequente nas organizações, o presenteísmo é um fenômeno investigado recentemente e em muitas situações pouco percebido, apesar de interferir negativamente na produtividade, no desempenho do trabalhador e na elevação dos custos. Além disso, é reconhecido como um dos fatores de risco para um futuro absenteísmo por doença (QUEIROZ-LIMA; SERRANHEIRA, 2016).

O estresse ocupacional e o presenteísmo são temas discutidos mundialmente e que têm repercussão em vários segmentos organizacionais, pois além das consequências à saúde do trabalhador, esses fenômenos também podem interferir na capacidade de cuidar e na segurança do paciente, com maior chance de erros relacionados às tarefas laborais executadas, expondo os pacientes a riscos (JEON et al., 2014; LY et al., 2019; KERSTEN; VINCENT-HÖPER; NIENHAUS, 2020).

Dessa forma, este estudo teve como objetivo a investigação do estresse ocupacional e do presenteísmo entre enfermeiros atuantes em um hospital público de alta complexidade e de referência regional. 


\section{METODOLOGIA}

Trata-se de um estudo quantitativo, descritivo, transversal, realizado em um hospital público de alta complexidade e de referência regional, localizado no município de Campos dos Goytacazes - RJ.

A amostra foi selecionada por conveniência, sendo incluídos os enfermeiros atuantes no hospital supracitado há mais de um ano. Foram excluídos os que, no momento da coleta de dados, estiveram de licença médica ou licença maternidade.

A coleta de dados foi realizada nos meses de junho a setembro de 2019, por meio de três instrumentos: um questionário para caracterização sociodemográfica e funcional, o Inventário de Estresse em Enfermeiros (IEE) e o Questionário de Limitações no Trabalho (WLQ). Os questionários foram entregues aos participantes, com retorno agendado segundo disponibilidade dos participantes.

As variáveis investigadas foram: idade, sexo, escolaridade, estado civil, paternidade/maternidade, realização de algum tratamento de saúde, carga horária semanal de trabalho, outro emprego, tempo de trabalho na enfermagem, turno de trabalho, faltas ao trabalho no último ano, motivos pela falta e número de faltas.

O IEE foi desenvolvido e validado para os indivíduos brasileiros e fornece uma medida geral de estresse ocupacional do enfermeiro a partir da identificação dos estressores e da frequência com que estes são percebidos nas atividades laborais. É composto por 38 itens, dispostos em uma escala Likert de cinco pontos, que variam de um, assinalado para opção nunca, a cinco, para a frequência sempre. Os itens são distribuídos em categorias, sendo: relações interpessoais (17 itens), papéis estressores da carreira (11 itens) e fatores intrínsecos ao trabalho (10 itens). Na avaliação do IEE, a média representa baixo ou alto estresse para a população, sendo que valores abaixo ou iguais a três são afirmativos para a primeira hipótese (baixo estresse) e acima de três para a segunda (alto estresse) no ambiente de trabalho (STACCIARINI; TRÓCCOLI, 2000).

O WLQ é um instrumento traduzido, adaptado culturalmente e validado para a realidade brasileira. Foi desenvolvido para avaliar o presenteísmo a partir da medida de produtividade perdida associada à interferência dos problemas de saúde no desempenho das atividades no trabalho. É composto por 25 itens, agrupados em um quadro denominado domínios de limitação do trabalho, que abrangem o caráter multidimensional das funções desenvolvidas no ambiente ocupacional, sendo: gerência de tempo (5 itens), demanda física (6 itens), demanda mental-interpessoal (9 itens) e demanda de produção (5 itens) (SOÁREZ et al., 2007).

Os dados coletados foram digitados no Excel e exportados para o Statistical Package for the Social Sciences (SPSS), versão 24.0, no qual foram realizadas as análises descritivas uni e bivariadas. As variáveis qualitativas foram descritas por meio da frequência absoluta e relativa; e as quantitativas, por meio de medidas descritivas (mínimo, máximo, média e desvio padrão).

O teste de correlação entre os escores do IEE e os índices do WLQ foi realizado pelo coeficiente de correlação de Spearman. A consistência interna das escalas foi avaliada pelo Coeficiente Alfa de Cronbach, a fim de se verificar a fidedignidade da medida a que os instrumentos se propõem a mensurar. Valores acima de 0,70 são considerados confirmativos para esse fim (FIELD, 2009).

Em consonância com as diretrizes e normas regulamentadoras de pesquisa envolvendo seres humanos, vigentes no período da realização desta pesquisa, a Resolução do CNS 466/12 (BRASIL, 2012), o projeto foi aprovado pelo Comitê de Ética em Pesquisa dos Institutos Superiores de Ensino do CENSA (ISECENSA) e aprovado sob o protocolo CAAE $n^{\circ} 13244418.5 .0000 .5524$. A participação dos sujeitos 
foi aceita mediante a exposição dos objetivos deste estudo, os esclarecimentos acerca da pesquisa e a assinatura do Termo de Consentimento Livre e Esclarecido (TCLE).

\section{RESULTADOS}

A amostra foi composta por 42 enfermeiros atuantes em um hospital público de alta complexidade. Em relação aos dados sociodemográficos, verificou-se o predomínio de enfermeiros do sexo feminino $(73,8 \%)$, casados $(57,1 \%)$, com filhos $(66,7 \%)$, pós-graduados $(64,3 \%)$ e que não estavam em tratamento de saúde no momento da coleta (75,6\%). A média de idade foi de 42,1 anos ( $d p=9,05$ anos).

Tabela 1 - Distribuição da amostra, segundo variáveis qualitativas. Campos dos Goytacazes/RJ, 2019.

\begin{tabular}{|c|c|c|}
\hline \multirow{2}{*}{ Variáveis qualitativas } & \multicolumn{2}{|c|}{ Frequência } \\
\hline & Absoluta (n) & Relativa (\%) \\
\hline \multicolumn{3}{|l|}{ Sexo } \\
\hline Feminino & 31 & 73,8 \\
\hline Masculino & 11 & 26,2 \\
\hline \multicolumn{3}{|l|}{ Situação conjugal } \\
\hline Casados & 24 & 57,1 \\
\hline Solteiros & 13 & 31,0 \\
\hline Divorciados & 5 & 11,9 \\
\hline \multicolumn{3}{|l|}{ Filhos } \\
\hline Sim & 28 & 66,7 \\
\hline Não & 14 & 33,3 \\
\hline \multicolumn{3}{|l|}{ Pós-graduação } \\
\hline $\operatorname{Sim}$ & 32 & 76,2 \\
\hline Não & 10 & 23,8 \\
\hline \multicolumn{3}{|l|}{ Tratamento de saúde } \\
\hline $\operatorname{Sim}$ & 10 & 24,4 \\
\hline Não & 31 & 75,6 \\
\hline \multicolumn{3}{|l|}{ Turno } \\
\hline Diurno & 7 & 16,7 \\
\hline Diurno e noturno $(24 \mathrm{H})$ & 35 & 83,3 \\
\hline \multicolumn{3}{|l|}{ Outro emprego } \\
\hline Sim & 35 & 83,3 \\
\hline Não & 7 & 16,7 \\
\hline \multicolumn{3}{|l|}{ Faltas ao trabalho } \\
\hline $\operatorname{Sim}$ & 10 & 23,8 \\
\hline Não & 32 & 76,2 \\
\hline \multicolumn{3}{|l|}{ Motivos das faltas } \\
\hline Doenças diagnosticadas & 5 & 50,0 \\
\hline Cirurgias diversas & 3 & 30,0 \\
\hline $\begin{array}{l}\text { Razões diversas de caráter } \\
\text { familiar }\end{array}$ & 2 & 20,0 \\
\hline
\end{tabular}

Fonte: Elaborada pelas autoras, 2019. 
Quanto à caracterização funcional da amostra, constatou-se que $83,3 \%$ dos enfermeiros trabaIham no turno diurno e noturno em esquema de vinte e quatro horas e têm outro emprego. No último ano, $23,8 \%$ relataram faltas ao trabalho e os motivos constam como doenças diagnosticadas (50\%). 0 número médio de faltas ao trabalho foi de 14,8 dias ( $\mathrm{dp}=11,1$ dias). A carga horária semanal média de trabalho foi de 35,8 horas ( $d p=13,5$ horas) e o tempo médio de atuação na enfermagem foi de 17,8 anos $(\mathrm{dp}=9,04$ anos).

Tabela 2 - Distribuição da amostra, segundo variáveis quantitativas. Campos dos Goytacazes/RJ, 2019.

\begin{tabular}{lcccc}
\hline \multirow{2}{*}{ Variáveis quantitativas } & \multicolumn{4}{c}{ Valores } \\
\cline { 2 - 5 } & Mínimo & Máximo & Média & $\begin{array}{c}\text { Desvio- } \\
\text { amostra }\end{array}$ \\
\hline Idade (anos) & 25 & 61 & 42,1 & 9,05 \\
\hline Número de faltas (dias) & 2 & 30 & 14,8 & 11,1 \\
\hline Carga horária semanal de trabalho (horas) & 24 & 64 & 35,8 & 13,5 \\
\hline Tempo de atuação na enfermagem (anos) & 8 & 38 & 17,8 & 9,04 \\
\hline
\end{tabular}

Fonte: Elaborada pelas autoras, 2019.

A consistência interna das escalas, avaliadas pelo Coeficiente Alfa de Cronbach, atestou fidedignidade aos instrumentos. 0 instrumento IEE obteve 0,80 de confiabilidade Alfa de Cronbach e o instrumento WLQ teve 0,96 de confiabilidade pelo mesmo instrumento de confiabilidade.

Os enfermeiros investigados neste estudo apresentaram alta intensidade de estresse, visto que a média geral da escala IEE foi de 3,57 ( $d p=0,41)$. Além disso, 92,7\% apresentaram valores para a média acima de três pontos na escala tipo Likert. Dentre as situações avaliadas pelo IEE, as relações interpessoais apresentaram a maior média, sendo de $3,80(\mathrm{dp}=0,55)$. Quanto ao instrumento $W L Q$, a análise do escore evidenciou um decréscimo de $35,9 \%$ na produtividade desses enfermeiros. 0 domínio demanda mental-interpessoal, que avalia a dificuldade em realizar tarefas cognitivas no trabalho e interagir com pessoas nesse ambiente apresentou o maior valor médio, sendo de $12,95(\mathrm{dp}=5,54)$. Isso significa que, nas duas últimas semanas, os enfermeiros tiveram limitação maior que $10 \%$ do seu tempo para realizar esse tipo de tarefa no seu trabalho.

Tabela 3 - Valores obtidos os questionários IEE e WLQ. Campos dos Goytacazes/RJ, 2019.

\begin{tabular}{lccc}
\hline \multirow{2}{*}{ Instrumentos/Domínios } & \multicolumn{3}{c}{ Escores } \\
\cline { 2 - 4 } & Mínimo & Máximo & Média (dp) \\
\hline IEE geral & 2,90 & 4,40 & $3,57(0,41)$ \\
\hline Relações interpessoais & 2,53 & 4,71 & $3,80(0,55)$ \\
\hline Papéis estressores da carreira & 2,30 & 4,30 & $3,17(0,57)$ \\
\hline Fatores intrínsecos ao trabalho & 2,80 & 4,90 & $3,74(0,55)$ \\
\hline WLQ & 9,00 & 83,00 & $35,94(13,36)$ \\
\hline Gerência de tempo & 0,0 & 20,00 & $6,78(3,32)$ \\
\hline Demanda física & 6,00 & 26,00 & $9,27(4,57)$ \\
\hline Demanda mental-interpessoal & 0,0 & 32,00 & $12,95(5,54)$ \\
\hline Demanda de produção & 1,00 & 18,00 & $6,80(2,71)$ \\
\hline
\end{tabular}

Fonte: Elaborada pelas autoras, 2019. 
A correlação entre o Inventário de Estresse em Enfermeiros (IEE) e o Questionário de Limitações no Trabalho (WLQ) apresentou nível de significância próximo de 0,05 entre os domínios "papéis estressores da carreira" e a "gerência de tempo"; entre os domínios "fatores intrínsecos ao trabalho" e a "demanda de produção"; e entre o "IEE geral" e a "demanda de produção". As análises bivariadas indicam correlações positivas fracas, conforme apresentado na tabela 4.

Tabela 4 - Correlação entre IEE x WLQ. Campos dos Goytacazes/RJ, 2019.

\begin{tabular}{lccccc}
\hline \multirow{2}{*}{ IEE/WLQ } & \multicolumn{5}{c}{ Coeficientes de Correlação de Spearman } \\
\cline { 2 - 6 } & $\begin{array}{c}\text { Gerência de } \\
\text { tempo }\end{array}$ & Demanda física & $\begin{array}{c}\text { Demanda } \\
\text { mental-interpessoal }\end{array}$ & $\begin{array}{c}\text { Demanda de } \\
\text { produção }\end{array}$ & WLQ \\
\hline Relações interpessoais & 0,061 & 0,093 & 0,053 & 0,020 & 0,087 \\
\hline Papéis estressores da carreira & $0,277 *$ & 0,197 & 0,172 & 0,245 & 0,208 \\
\hline Fatores intrínsecos ao trabalho & 0,248 & 0,122 & 0,150 & $0,282 *$ & 0,173 \\
\hline IEE geral & 0,254 & 0,152 & 0,174 & $0,268^{* *}$ & 0,207 \\
\hline
\end{tabular}

*Nível de significância 0,07

**Nível de significância 0,09

Fonte: Elaborada pelas autoras, 2019

\section{DISCUSSÃo}

Os dados referentes à caracterização da amostra evidenciam o predomínio de enfermeiros do sexo feminino, casados e com filhos, corroborando com os dados encontrados em estudos nacionais (MACHADO et al., 2018; CARVALHO et al., 2020) e internacionais (RUIZ-FERNÁDEZ et al., 2020; KERSTEN; VINCENT-HÖPER; NIENHAUS, 2020).

Estudo realizado em um hospital universitário brasileiro identificou prevalências significativas entre essas variáveis e o estresse ocupacional, sendo as mulheres, os casados e aqueles com filhos os mais propensos. Sugere-se a influência de fatores externos ao trabalho, tais como fatores relativos à vida pessoal e familiar do indivíduo, sob o estresse ocupacional (PEREIRA-FERREIRA; AZEVEDO; ROCHA, 2019). A média de idade foi acima de 40 anos e, por conseguinte, um tempo médio de atuação na enfermagem superior a 15 anos. Contrapondo a esse dado, os autores supracitados indicam que os primeiros cinco anos de trabalho em um hospital é o período de tempo com maior probabilidade de desenvolvimento do estresse ocupacional para esses profissionais. A hipótese é de que o turno diurno e noturno em esquema de vinte e quatro horas e o fato de possuírem outro emprego contribuem para a intensidade de estresse presente na amostra.

A utilização do IEE permitiu investigar o estresse entre os sujeitos desta pesquisa. Os participantes obtiveram pontuações condizentes com um alto nível de estresse ocupacional. Um estudo realizado com essa categoria profissional em um hospital do interior do Rio Grande do Sul apresentou resultado semelhante, sendo o elevado nível de estresse ocupacional atribuído ao contínuo contato com pacientes críticos, exposição prolongada a situações difíceis e excessiva carga de trabalho (UMANN; GUIDO; SILVA, 2014). Outro estudo realizado por Antoniolli et al. (2017) sobre o estresse entre enfermeiros e equipe de enfermagem evidenciou alto nível de estresse, corroborando com os resultados encontrados nesta pesquisa.

Dos três fatores específicos do inventário, o referente a relações interpessoais que pondera sobre as relações no ambiente de trabalho com outros profissionais, com pacientes e familiares, com alunos, 
com o grupo de trabalho e também com a própria família do profissional foi o mais prevalente. Nessa perspectiva, admite-se que o apoio social entre os profissionais enfermeiros, dos chefes e da equipe de enfermagem é essencial nas relações de trabalho. 0 gerenciamento de pessoas, uma das atribuições dos enfermeiros, demanda maior tempo de trabalho e atenção dedicada à interação, aumentando a probabilidade de estresse por conflitos interpessoais. Considera-se que, em resposta às pressões vividas no exercício do trabalho, relacionamentos menos humanos e mais técnicos são estabelecidos, contribuindo para o estresse ocupacional (UMANN; GUIDO; SILVA, 2014). Esse fator tem sido apontado como o principal causador de estresse entre essa categoria, sendo a interação dos enfermeiros com os pacientes e com os outros profissionais, devido à falta de confiança e à competitividade, importantes causas de estresse (ANTONIOLLI et al., 2017).

Para a investigação a respeito do presenteísmo, a aplicação do instrumento WLQ apontou um decréscimo significativo na produtividade. Essa perda resulta em consequências tanto para a organização do serviço e instituição, quanto para os profissionais e para a assistência prestada (UMANN; GUIDO; SILVA, 2014). O domínio que representou maior limitação para essa amostra foi a demanda mental-interpessoal. Esse resultado sugere que a dificuldade para realizar tarefas cognitivas e para interagir com as pessoas no trabalho influencia a perda da produtividade desses enfermeiros, sendo uma limitação superior a 10\% do seu tempo para realizar essas atividades, nas últimas duas semanas. Segundo Li et al. (2019), o presenteísmo dos enfermeiros causa um declínio em sua saúde física e mental e no desempenho no trabalho, resultando em perda de produtividade e aumento dos custos para as organizações.

Neste estudo, investigou-se a correlação entre o estresse e o presenteísmo identificados pelos instrumentos IEE e WLQ, respectivamente. 0 teste de correlação aplicado apresentou nível de significância próximo de 0,05 apenas entre algumas dimensões, indicando uma correlação positiva fraca. Estudo realizado por Umann, Guido e Silva (2014) apontou correlações diretas e significativas entre os escores dessas duas escalas. A hipótese é de que o tamanho da amostra e o tipo de instituição investigada tenham influenciado as análises de correlação entre as escalas.

Contudo, a saúde e a capacidade para o trabalho são, em partes, determinadas pela forma como o indivíduo se adapta à atividade laboral e pelo grau de tolerância ao trabalho, que pode ser determinado pelas características individuais e pela inter-relação entre as condições de vida e de trabalho de cada indivíduo. Assim sendo, as altas demandas de trabalho, o estresse e o menor controle sobre as atividades constituem fatores preditores do presenteísmo, resultando em maior perda da produtividade (JEON et al., 2014).

\section{CONCLUSÃO}

0 alto nível de estresse ocupacional esteve presente na maioria dos enfermeiros investigados. Dentre as dimensões avaliadas pelo IEE, a dimensão relações interpessoais foi a mais prevalente, indicando maior influência das relações no ambiente de trabalho e também com a própria família no nível de estresse desses profissionais.

Observou-se uma perda de $35,9 \%$ na produtividade, sendo o domínio demanda mental-interpessoal o de maior valor médio, evidenciando a dificuldade desses enfermeiros para realizar tarefas cognitivas e interagir com pessoas no trabalho. Esses resultados indicam que o estresse influencia inversamente a produtividade, considerando também os fatores organizacionais e/ou individuais frente às circunstâncias que envolvem suas atividades laborais.

Considera-se que o bom desempenho profissional, bem como uma produtividade adequada às demandas de trabalho são alcançados mediante esforço individual e suporte organizacional. Assim sendo, 
sugere-se a implementação de estratégias que busquem desenvolver a capacidade de interação no ambiente de trabalho, contribuindo com a qualidade de vida no trabalho e a qualidade da assistência prestada.

Este estudo teve como limitação o tamanho da amostra e o tipo de instituição investigada. Por se tratar de um hospital público, os profissionais possuem estabilidade de vínculo, o que permite 0 afastamento para recuperação da saúde, limitando a avaliação do presenteísmo.

Além disso, o estresse é considerado um fenômeno dinâmico e a forma como os indivíduos percebem seu ambiente de trabalho pode mudar ao longo do tempo. Desse modo, o delineamento transversal utilizado retrata a percepção atual desses profissionais no contexto vivenciado no período em que os dados foram coletados. Ressalta-se também a necessidade de se comparar os diferentes setores do hospital em estudo futuro, a fim de se considerar as atividades e as demandas de cada setor.

\section{REFERÊNCIAS}

ANTONIOLLI, L.; ECHEVARRÍA-GUANILO, M.E.; MARTINS, C.L.; AMESTOY, S.C.; LONGARAY, T.M.; DESOUZA, S.B.C. Coping e estresse na equipe de enfermagem de um centro de tratamento de queimados. Rev. Bras. Queimaduras, v. 16, n. 3, p. 174-180, 2017. Disponível em: https://lume.ufrgs.br/bitstream/ handle/10183/212334/001114893.pdf? sequence=1\&isAllowed=y. Acesso em: 24 mar. 2020.

CARVALHO, A.E.L.; FRAZÃO, I.S.; SILVA, D.M.R.; ANDRADE, M.S.; VASCONCELOS, S.C.; AQUINO, J.M. Estresse dos profissionais de enfermagem atuantes no atendimento pré-hospitalar. Rev. Bras. Enfermagem, v. 73, n. 2, p. e20180660, 2020. Disponível em: https://www.scielo.br/scielo.php?script=sci_arttext\&pid=S003471672020000200173\&tlng=pt. Acesso em: 15 mar. 2020.

CONSELHO NACIONAL DE SAÚDE (Brasil). Resolução n .466 de 12 de dezembro de 2012. Ministério da Saúde. Brasília: 2012. Disponível em: http://conselho.saude.gov.br/resolucoes/2012/Reso466.pdf. Acesso em: 15 mar. 2019.

CUNHA, I.C.K.O.; BALSANELLI, A.P. Liderança do enfermeiro em unidade de terapia intensiva e sua relação com ambiente de trabalho. Rev. Latino-Am. Enfermagem, v. 23, n. 1, p. 106-113, 2015. Disponível em: https://www.scielo.br/pdf/rlae/v23n1/pt_0104-1169-rlae-23-01-00106.pdf. Acesso em: 15 set. 2019.

FELIX, D. B.; MACHADO, D. Q.; SOUSA, E. F.; CARNEIRO, J. V. C. Análise dos níveis de estresse no ambiente hospitalar: um estudo com profissionais da área de enfermagem. Revista de Carreiras e Pessoas, v. 7, n. 2, p. 530-543, 2017. Disponível em: https://revistas.pucsp.br/index.php/ReCaPe/article/view/32749/22616. Acesso em: 23 mar. 2019.

FIELD, A. Descobrindo a estatística usando o SPSS. 2. ed. Porto Alegre: Artmed, 2009.

JEON, S.H.; LEEM, J.H.; PARK, S.G.; HEO, Y.S.; LEE, B.J.; MOON, S.H.; JUNG, D.Y.; KIM, H.C. Association among working hours, occupational stress, and presenteeism among wage workers: results from the second korean working conditions survey. Ann. Occup. Environ. Med., v. 26, n. 1, p. 6-13, 2014. Disponível em: https://www.ncbi.nlm.nih.gov/pmc/articles/PMC3994451/. Acesso em: 24 mar. 2019.

KERSTEN, M.; VINCENT-HÖPER, S.; NIENHAUS, A. Stress of dialysis nurses-analyzing the buffering role of influence at work and feedback. Int. J. Environ Res Public Health, v. 17, n. 3, p. E802, 2020. Disponível em: https://www.ncbi.nlm.nih.gov/pmc/articles/PMC7036795. Acesso em: 23 mar. 2019. 
LI, Y.; ZHANG, J.; WANG, S.; GUO, S. The effect of presenteeism on productivity loss in nurses: the mediation of health and the moderation of general self-efficacy. Frente. Psychol., v. 10, p. 1745, 2019. Disponível em: https://www.ncbi.nlm.nih.gov/pmc/articles/PMC6685003/. Acesso em: 24 mar. 2019.

MACHADO, D.A.; FIGUEIREDO, N.M.A.; VELASQUES, L.S.; BENTO, C.A.M.; MACHADO, W.C.A.; VIANNA, L.A.M. Cognitive changes in nurses working in intensive care units. Rev. Bra. Enfermagem, v. 71, n. 1, p. 73-9, 2018. Disponível em: https://www.scielo.br/scielo.php?script=sci_arttext\&pid=S0034-71672018000100073. Acesso em: 16 mar. 2020.

OLIVEIRA, E.B.; GALLASH, C.H.; JUNIOR, P.P.A.S.; OLIVEIRA, A.V.R.; VALÉRIO, R.L.; DIAS, L.B.S. Occupational stress and burnout in nurses of an emergency service: the organization of work. Rev. Enferm. UERJ, v. 25, p. e28842, 2017. Disponível em: https://www.e-publicacoes.uerj.br/index.php/enfermagemuerj/ article/view/28842/22382. Acesso em: 16 mar. 2020.

PEREIRA-FERREIRA, J. M.; AZEVEDO, A. R. I.; ROCHA, M. S. Análise do estresse ocupacional em funcionários de um hospital universitário. Revista de Carreiras e Pessoas, v. 9, n. 3, p. 295-314, 2019. Disponível em: https://revistas.pucsp.br/index.php/ReCaPe/article/view/41218/29585. Acesso em: 23 mar. 2019.

QUEIROZ-LIMA, M.E.; SERRANHEIRA, F. Absenteeism and presenteeism costs from occupational accidents with WRMSDs in a Portuguese hospital. Dyna rev.fac.nac.minas, Medellín, v. 83, n. 196, p. 27-30, 2016. Disponível em: http://www.scielo.org.co/scielo.php?script=sci_arttext\&pid=S0012-73532016000200004. Acesso em: 23 mar. 2020.

RUIZ-FERNÁNDEZ, M.D.; ORTEGA-GALÁN, A.M.; FERNÁNDEZ-SOLA, C.; HERNÁNDEZ-PADILLA, J.M.; GRANERO-MOLINA, J.; RAMOS-PICHARDO, J.D. Occupational factors associated with health-related quality of lefi in nursing professionals: a multicentre study. Int. J. Environ. Res. Public Health, v. 17, n. 3, p. 982, 2020. Disponível em: https://www.ncbi.nlm.nih.gov/pmc/articles/PMC7038014/. Acesso em: 23 mar. 2019.

SANTOS, N.A.R.; SANTOS, J.; SILVA, V.R.; PASSOS, J.P. Occupational stress in palliative care in oncology. Cogitare Enferm., v. 22, n. 4, p. e50686, 2017. Disponível em: https://docs.bvsalud.org/ biblioref/2017/12/876588/50686-219741-1-pb.pdf. Acesso em: 16 mar. 2020.

SKAGEN, K.; COLLINS, A.M. The consequences of sickness presenteeism on health and wellbeing over time: a systematic review. Soc. Sci. Med., v. 161, p. 169 - 177, 2016. Disponível em: https://www. sciencedirect.com/science/article/abs/pii/S0277953616302866?via\%3Dihub. Acesso em: 16 mar. 2020.

SOÁREZ, P.C.; KOWALSKI, C.C.G.; FERRAZ, M.B.; CICONELLI, R.M. Tradução para português brasileiro e validação de um questionário de avaliação de produtividade. Revista Panamericana Salud Publica, v. 22, p. 21-28, 2007. Disponível em: https://iris.paho.org/bitstream/handle/10665.2/7851/a03v22n1. pdf?sequence=1\&isAllowed=y. Acesso em: 18 nov. 2018.

STACCIARINI, J.M.R.; TRÓCCOLI, B.T. Instrumento para mensurar o estresse ocupacional: inventario de estresse em enfermeiros (IEE). Rev. Latino Am. Enfermagem, v. 8, n. 60, p. 40-49, 2000. Disponível em: https://www.scielo.br/pdf/rlae/v8n6/12347.pdf. Acesso em: 18 nov. 2018.

UENO, L.G.S.; BOBROFF, M.C.C.; MARTINS, J.T.; MACHADO, R.C.B.R.; LINARES, P.G.; GASPAR, S.G. Occupational stress: stressors referred by the nursing team. J. Nurs. UFPE On Line, v. 11, n. 4, p. 1632-1638, 2017. Disponível em: https://periodicos.ufpe.br/revistas/revistaenfermagem/article/ view/15232/18001. Acesso em: 16 mar. 2020. 
UMANN, J.; GUIDO, L.A.; SILVA, R.M. Estresse, coping e presenteísmo em enfermeiros que assistem pacientes críticos e potencialmente críticos. Rev. Esc. Enferm. USP, v. 48, n. 5, p. 891-898, 2014. Disponível em: https://www.scielo.br/pdf/reeusp/v48n5/pt_0080-6234-reeusp-48-05-891.pdf. Acesso em: 18 nov. 2018. 